\title{
ON $S$-REDUCIBLE FINSLER SPACES
}

\author{
M. VERMA, B. N. PRASAD AND R. K. PANDEY
}

\section{Introduction}

To study the theory of Fields in a Finsler space $F_{n}$, it is important to introduced the Ricci tensors of $F_{n}$. The Ricci tensor

$$
\mathbb{R}_{i j}=\mathbb{R}_{h i j k} g^{h k}
$$

of a Riemanian space plays the essential role in the theory of graviation. But we have very few papers ([8], [9]) concern with such tensors of $(0,2)$ type constructed from the curvature tensors of $F_{n}$.

For the $v$-curvature tensor $S_{h i j k}$ of $F_{n}$, the problem is interesting because it is regarded as the Riemanian curvature tensor of the tangent Riemanian space of $F_{n}$. The $v$-Ricci tensor of $F_{n}$ is defined from $v$-curvature tensor $S_{h i j k}$ as

$$
S_{i j}=S_{h i j k} g^{h k}
$$

This tensor is symmetric and indicatory tensor. In this paper we shall study those Finsler spaces for which

$$
S_{i j}=\rho h_{i j}+\mu C_{i} C_{j}
$$

where $\rho$ and $\mu$ are scalar functions, $h_{i j}$ are components of angular metric tensor and $C_{i}$ are components of contracted $C$-tensor i.e.

$$
C_{i}=C_{i j}^{j}
$$

Received July 20, 1991. 
The $v$-Ricci tensor of $C$-reducible and semi $C$-reducible Finsler space is of this form. So we shall say the Finsler space whose $v$-Ricci tensor is of the form (1.3) as $S$-reducible Finsler space.

There are three kinds of torsion tensors in Cartan's theory of Finsler space $F_{n}$. Two of them are $(h) h v$-torsion tensor $C_{i j k}$ and $(v) h v$-torsion tensor $P_{i j k}$ which are symmetric in all of its indices. Since the $v$-curvature tensor and $v$ Ricci tensor are defined from the $(h) h v$-torsion tensor $C_{j k}^{j}$. in cartan's theory of Finsler spaces as

$$
\begin{aligned}
S_{h j k}^{i} & =\partial_{k} C_{h j}^{i}+C_{h j}^{r} C_{r k}^{i}-\partial_{j} C_{h k}^{i}-C_{h k}^{r} C_{r j}^{i} \\
& =C_{h k}^{r} C_{r j}^{i}-C_{h j}^{r} C_{r k}^{i}
\end{aligned}
$$

Therefore, the forms of $v$-Ricci tensor of Finsler space will depend upon the special form of $C_{i j k}$. Many workers have obtained interesting forms of $C_{i j k}$. They are $C$-reducible ([3]):

$$
C_{i j k}=\frac{1}{n+1}\left(h_{i j} C_{k}+h_{j k} C_{i}+h_{k i} C_{j}\right)
$$

Semi C-reducible ([4]):

$$
C_{i j k}=\frac{p}{n+1}\left(h_{i j} C_{k}+h_{j k} C_{i}+h_{k i} C_{j}\right)+\frac{q}{C^{2}} C_{i} C_{j} C_{k}
$$

$C_{2}$-like ([5]):

$$
C_{i j k}=\frac{1}{C^{2}} C_{i} C_{j} C_{k}
$$

where $p$ and $q$ in (1.7) are scalars satisfying $p+q=1$.

The S3- and S4- like Finsler spaces are characterized by relations ([2], [4])

$$
\begin{aligned}
& L^{2} S_{h i j k}=S\left(h_{h j} h_{i k}-h_{h k} h_{i j}\right), \quad n \geq 4 \\
& L^{2} S_{h i j k}=h_{h j} M_{i k}+h_{i k} M_{h j}-h_{h k} M_{i j}-h_{i j} M_{h k}
\end{aligned}
$$

respectively. Where $S$ is $(0) p$-homogeneous and $M_{i j}$ is symmetric and indicatory tensor. 
The various transformations of a Finsler metric have been studied in the literature ([1]). We shall also study the conditions under which the $S$-reducible Finsler space is transformed to $S$-reducible Finsler space under these transformations of the metric.

\section{The $v$-Ricci Tensor of Special Finsler Spaces}

The $v$-curvature tensor of $C$-reducible Finsler space is given by ([3])

$$
S_{i j k l}=\frac{1}{(n+1)^{2}}\left(h_{i l} C_{j k}+h_{j k} C_{i l}-h_{i k} C_{i l}-h_{j l} C_{i k}\right)
$$

where $C_{i j}=\frac{1}{2} C^{r} C_{r} h_{i j}+C_{i} C_{j}$

With help of equations (1.2) and (2.1), we have

$$
S_{j k}=\rho h_{j k}+\mu C_{j} C_{k}
$$

where $\rho=\frac{n-1}{(n+1)^{2}} C^{2}$ and $\mu=\frac{n-3}{(n+1)^{2}}$

Therefore, we have the

Theorem 2.1. Every C-reducible Finsler space is S-reducible Finsler space.

The v-curvature tensor for semi $C$-reducible Finsler space is given as ([4])

$$
\mathbb{L}^{2} S_{h i j k}=h_{h j} M_{i k}+h_{i k} M_{h j}-h_{h k} M_{i j}-h_{i j} M_{h k}
$$

where

$$
M_{i j}=-\mathbb{L}^{2} \frac{p^{2} c^{2}}{2(n+1)^{2}} h_{i j}-\mathbb{L}^{2}\left\{\frac{p^{2}}{(n+1)^{2}}+\frac{p q}{n+1}\right\} C_{i} C_{j}
$$

In view of equations (1.2) and (2.2), the $v$-Ricci tensor for semi $C$-reducible Finsler space can be written as

$$
S_{i j}=\rho h_{i j}+\mu C_{i} C_{j}
$$

where

$$
\rho=\left\{\frac{(n-3) p^{2} C^{2}}{2(n+1)^{2}}-\frac{p C^{2}}{n+1}\left(\frac{p}{2}+q\right)\right\}
$$

and 


$$
\mu=(n-3)\left\{\frac{p^{2}}{(n+1)^{2}}+\frac{p q}{n+1}\right\}
$$

hence, we have the following.

Theorem 2.2. Every semi $C$-reducible Finsler space is S-reducible Finsler space.

The $v$-curvature tensor of $S 3$-like Finsler space is written as (1.9). In view of equations (1.2) and (1.9), the $v$-Ricci tensor for S3-like Finsler space can be written as

$$
S_{i j}=\rho h_{i j}+\mu C_{i} C_{j}
$$

where $\rho=-\frac{S}{L^{2}}(n-2)$ and $\mu=0$

Therefore, we have

Theorem 2.3. Every S3-like Finsler space is S-reducible Finsler space.

The v-curvature tensor for $S 4$-like Finsler space is given by (1.10). In view of equations (1.2) and (1.10), the $v$-Ricci tensor for $S 4$-like Finsler space can be written as

$$
S_{i j}=\rho h_{i j}+\mu M_{i j}
$$

where $\rho=-\frac{M_{h k} g^{h k}}{L^{2}}$ and $\mu=-\left(\frac{n-3}{L^{2}}\right)$

Therefore, we have the followings:

Theorem 2.4. The $v$-Ricci tensor of S4-like Finsler space is of the form

$$
S_{i j}=\rho h_{i j}+\mu M_{i j}
$$

and

Theorem 2.5. The S4-like Finsler space is S-reducible if and only if there exits scalars $\alpha$ and $\beta$ with $\mu \alpha+\rho \neq 0$ such that

$$
M_{i j}=\alpha h_{i j}+\beta C_{i} C_{j}
$$


In view of equations (1.8) and (1.5), we have

$$
S_{h i j k}=0
$$

which gives

$$
S_{i j}=0
$$

Thus, we have

Theorem 2.6. The v-Ricci tensor of C2-like Finsler space vanishes identically.

\section{The Transformation of $S$-Reducible Finsler Space by an $h$-Vector}

Prasad and Srivastave ([7]) obtained the relation between $v$-curvature tensors with respect to $C \Gamma$ of a Finsler space $\left(F_{n}, L\right)$ and $\left(F_{n}, L^{*}\right)$, where $\mathbb{L}^{*}(x, y)$ is obtained from $L(x, y)$ by the transformation

$$
\mathbb{L}^{* 2}(x, y)=\mathbb{L}^{2}(x, y)+\left(b_{i} y^{i}\right)^{2}
$$

where $b_{i}(x, y)$ is an $h$-vector in $\left(F_{n}, L\right)$.

The $v$-curvature tensor has been given as

$$
S_{h i j k}^{*}=\sigma S_{h i j k}+h_{i j} d_{h k}+h_{h k} d_{i j}-h_{i k} d_{h j}-h_{h j} d_{i k}
$$

where $d_{i j}=\frac{1}{2} \alpha_{1} h_{i j}+\alpha_{2} m_{i} m_{j}$ and $\alpha_{1}$ and $\alpha_{2}$ are scalar functions

$$
m_{i}=b_{i}-\frac{b_{r} y^{r}}{L} l_{i}
$$

The contravariant component of fundamental metric tensor $g^{* i j}$ of $\left(F_{n}, L^{*}\right)$ has been given by

$$
g^{* i j}=\sigma^{-1} g^{i j}-\frac{(1-\sigma) \beta}{L \lambda}\left(l^{i} b^{j}+l^{j} b^{i}\right)+\frac{(1-\sigma)\left(b^{2}+\sigma\right)}{\lambda} l^{i} l^{j}+\frac{1}{\lambda} b^{i} b^{j}
$$


where $\sigma=\left(1+\frac{\beta \rho}{L}\right), b^{2}=b^{i} b_{i}, \beta=b_{i} y^{i}$ and $\rho=\frac{1}{n-1} L C^{i} b_{i}$. The $(h) h v$-torsion tensor has been given by

$$
\begin{aligned}
C_{i j}^{* h}= & C_{i j}^{h}+\frac{\rho}{2 L \sigma}\left(h_{i j} m^{h}+h_{j}^{h} m_{i}+h_{i}^{h} m_{j}\right) \\
& -\frac{(1-\sigma) \beta \rho}{L^{2} \lambda}\left[\left\{\sigma+\frac{1}{2}\left(b^{2}-\frac{\beta^{2}}{L^{2}}\right)\right\} h_{i j l}^{h}+m_{i} m_{j} l^{h}\right] \\
& +\frac{\rho}{L \lambda}\left[\left\{\sigma+\frac{1}{2}\left(b^{2}-\frac{\beta^{2}}{L^{2}}\right)\right\} h_{i j} b^{h}+m_{i} m_{j} b^{h}\right]
\end{aligned}
$$

The angular metric tensor $h_{i j}^{*}$ of $\left(F_{n}, L^{*}\right)$ has been given by

$$
h_{i j}^{*}+l_{i}^{*} l_{j}^{*}=\sigma h_{i j}+\ell_{i} l_{j}+b_{i} b_{j}
$$

where

$$
L^{*} l_{i}^{*}=L l_{i}+\beta b_{i}
$$

The $v$-Ricci tensor of $\left(F_{n}, L^{*}\right)$ is obtained from equations (3.2), (3.3) and (1.3), which is given by

$$
S_{i j}^{*}=A h_{i j}+\mu C_{i} C_{j}+B m_{i} m_{j}
$$

where

and

$$
\begin{aligned}
A & =\left[\rho+\frac{1}{\sigma} M+\frac{1}{\lambda} N\left(b^{2}-\frac{\beta^{2}}{L^{2}}\right)+\frac{1}{2} \alpha_{1}\left\{\frac{1}{\sigma}(n-3)+\frac{1}{\lambda}\left(b^{2}-\frac{\beta^{2}}{L^{2}}\right)\right\}\right], \\
M & =\frac{1}{2}(n-1) \alpha_{1}+\alpha_{2}\left(b^{2}-\frac{\dot{\beta}}{L^{2}}\right), N=\frac{1}{2}+\alpha_{2}\left(b^{2}-\frac{\beta^{2}}{L^{2}}\right)
\end{aligned}
$$

$$
B=\alpha_{2}\left\{\frac{1}{\sigma}(n-3)+\frac{1}{\lambda}\left(b^{2}-\frac{\beta^{2}}{L^{2}}\right)\right\}-\frac{2}{\lambda}
$$

In view of equations (1.4), (3.4), we get

$$
C_{i} C_{j}=C_{i}^{*} C_{j}^{*}-M\left(m_{i} C_{j}+m_{j} C_{i}\right)-M^{2} m_{i} m_{j}
$$

With the help of equations (3.6), (3.5) and (3.7), we have

$$
\begin{aligned}
S_{i j}^{* *}= & \rho^{*} h_{i j}^{*}+\mu^{*} C_{i}^{*} C_{j}^{*}+\frac{A}{\sigma}\left\{\left(\frac{L^{2}}{L^{* 2}}-1\right) l_{i} l_{j}+\frac{L \beta}{L^{* 2}}\left(l_{i} b_{j}+l_{j} b_{i}\right)+\left(\frac{\beta^{2}}{L^{* 2}}-1\right) b_{i} b_{j}\right\} \\
& -\mu M\left(m_{i} C_{j}+m_{j} C_{i}\right)+\left(B-\mu M^{2}\right) m_{i} m_{j}
\end{aligned}
$$


where $\rho^{*}=\frac{A}{\sigma}$ and $\mu^{*}=\mu$. Thus we have the following

Theorem 3.1. An S-reducibl Finsler space is transformed to an $S$-reducible Finsler space under the transformation (3.1) if and only if

$$
\begin{aligned}
& \frac{A}{\sigma}\left\{\left(\frac{L^{2}}{L^{* 2}}-1\right) l_{i} l_{j}+\frac{L^{\beta}}{L^{* 2}}\left(l_{i} b_{j}+l_{j} b_{i}\right)+\left(\frac{\beta^{2}}{L^{* 2}}-1\right) b_{i} b_{j}\right\} \\
& \quad+\mu M\left(m_{i} C_{j}+m_{j} C_{i}\right)+\left(B-\mu M^{2}\right) m_{i} m_{j}=0 .
\end{aligned}
$$

4. The transformation of $S$-reducible Finsler space by cubic transformation

Prasad, B. N. and Singh, J. N. ([6]) obtained the relation between $v$-curvature tensors of $\left(F_{n}, L\right)$ and $\left(F_{n}, L^{*}\right)$ by the transformation

$$
\mathbb{L}^{* 3}=\mathbb{L}^{3}+\beta^{3}
$$

where $\beta=b_{1} y^{i}, b_{i}(x)$ is a component of a covariant vector which is a function of position alone. They also obtained the angular metric tensor $h_{i j}^{*}$ of $\left(F_{n}, L^{*}\right)$ as

$$
h_{i j}^{*}=p h_{i j}+2 p q m_{1} m_{j}
$$

where $p=L L^{*-1}, q=\beta L^{*-1}, m_{i}=q l_{i}-p b_{i}$ and $l_{i}=\partial_{i} L$. The contravariant component of fundamental metric tensor $g^{* i j}$ of $\left(F_{n}, L^{*}\right)$ has been given by

$$
g^{* i j}=p^{-1} g^{i j}-p q^{3} \lambda\left(p+q b^{2}\right) l^{i} l^{j}+q^{2} \lambda\left(l^{i} b^{j}+l^{j} b^{i}\right)-2 p q \lambda b^{i} b^{j}
$$

where $b^{i}=g^{i j} b_{j}, l^{i}=g^{i j} l_{j}, b^{2}=g^{i j} b_{i} b_{j}$

and $\lambda^{-1}=p^{3}-q^{3}+2 p^{2} q b^{2}$.

The $(h) h v$-torsion tensor $C_{i j}^{* h}$ of $\left(F_{n}, L^{*}\right)$ has been given by

$$
\begin{aligned}
C_{i j}^{* h}= & C_{i j}^{h}+\frac{q^{2}}{2 L}\left(h_{i}^{h} m_{j}+h_{j}^{h} m_{i}\right)+p q \lambda\left(q l^{h}-2 p b^{h}\right) C_{i j} \\
& +p^{2} q^{3}\left(p+q b^{2}\right) \frac{\lambda}{2 L} h_{i j} b^{h}+\frac{p \lambda}{L} m_{i} m_{j} b^{h}
\end{aligned}
$$


where $C_{i j}=C_{i j k} b^{k}$. By using the above relation they gave the relation between $v$-curvature tensors of $\left(F_{n}, L\right)$ and $\left(F_{n}, L^{*}\right)$ as

$$
\begin{aligned}
S_{h i j k}^{*}= & \mathbb{P} S_{h i j k}+C_{i j} d_{h k}+C_{h k} d_{i j}-C_{i k} d_{h j} \\
& -C_{h j} d_{i k}+h_{i j} E_{h k}+h_{h k} E_{i j}-h_{i k} E_{h j}-h_{h j} E_{i k}
\end{aligned}
$$

where

$$
d_{h k}=\frac{p^{2} \lambda}{L} m_{h} m_{k}-p q^{3} C_{h k}-\frac{p^{2} q^{2} \lambda}{4 L} h_{h k}
$$

and

$$
E_{n k}=\frac{p q^{2} \lambda}{4 L^{2}}\left[q^{2}\left(2 p^{3}+1\right)-2 p^{5} b^{2}\right] m_{h} m_{k}-\frac{p q^{4} \lambda}{8 L^{2}}\left(q^{2}-p^{2} b^{2}\right) h_{h k}-\frac{p^{2} q^{2} \lambda}{4 L} C_{h k}
$$

In view of equation (1.2), the $v$-Ricci tensor $S_{i j}^{*}$ of $\left(F_{n}, L^{*}\right)$ is obtained with the help of equations (4.3), (4.5) and (1.3) as

$$
\begin{aligned}
S_{i j}^{*}= & {\left[\rho\left(\frac{1}{p} C_{m} b^{m}-2 p q \lambda C_{h k} b^{h} b^{k}\right) \frac{p^{2} q^{2} \lambda}{4 L}\right.} \\
& -\left\{\frac{1}{p}(n-3)-2 p q \lambda h_{h k} b^{h} b^{k}\right\}\left(q^{2}-p^{2} b^{2}\right)\left(\frac{p q^{4} \lambda}{8 L^{2}}\right) \\
& \left.+\left(\frac{1}{p} E_{h k} g^{h k}-2 p q \lambda E_{h k} b^{h} b^{k}\right)\right] h_{i j}+\mu C_{i} C_{j} \\
& +\left(\frac{1}{p} C_{m} b^{m}-2 p q \lambda C_{h k} b^{h} b^{k}\right)\left(\frac{p^{2} \lambda}{L} m_{i} m_{j}-p q^{3} C_{i j}\right) \\
& +\left\{\frac{1}{p}(n-3)-2 p q \lambda h_{h k} b^{h} b^{k}\right\}\left[\frac{p q^{2} \lambda}{4 L^{2}}\left\{q^{2}\left(2 p^{3}+1\right)-2 p^{5} b^{2}\right\}\right. \\
& \left.m_{i} m_{j}-\frac{p^{2} q^{2} \lambda}{4 L} C_{i j}\right]+\frac{1}{p}\left(C_{i j} g^{h k} d_{h k}-C_{i m}^{h} b^{m} d_{h j}\right. \\
& \left.-C_{j m}^{k} b^{m} d_{i k}\right)-2 p q \lambda\left(p S_{h i j k}+C_{i j} d_{h k}-C_{i k} d_{h j}\right. \\
& \left.-C_{h j} d_{i k}-h_{i k} E_{h j}-h_{h j} E_{i k}\right) b^{h} b^{k}
\end{aligned}
$$

In view of equations (1.4) and (4.4), we get 


$$
\begin{aligned}
C_{i} C_{j}= & C_{i}^{*} C_{j}^{*}+M\left(m_{i} C_{j}+m_{j} C_{i}\right) \\
& +2 p^{2} q \lambda\left(C_{i} C_{j}+C_{j} C_{i .}\right)-M^{2} m_{i} m_{j} \\
& -2 p^{2} q \lambda M\left(m_{i} C_{j .}+m_{j} C_{i .}\right)-4 p^{4} q^{2} \lambda C_{i .} C_{j} .
\end{aligned}
$$

where

$$
M=-\left\{\left(p^{-1}-n+1\right) \frac{q^{2}}{2 L}-\frac{q^{2}}{2 L}+\frac{p^{2} \lambda}{L}\left(b^{2}-\beta^{2} / L^{2}\right)\right\}
$$

and

$$
C_{i .}=C_{i j} b^{j}
$$

Using equations (4.2) and (4.7), the equation (4.6) can be written as

$$
S_{i j}^{*}=\rho^{*} h_{i j}^{*}+\mu^{*} C_{i}^{*} C_{j}^{*}+A_{i j}^{*}
$$

where

$$
\begin{aligned}
\rho^{*}= & \frac{1}{\rho}\left[\rho-\left(\frac{1}{p} C_{m} b^{m}-2 p q \lambda C_{h k} b^{h} b^{k}\right) \frac{p^{2} q^{2} \lambda}{4 L}\right. \\
& -\left\{\frac{1}{p}(n-3)-2 p q \lambda h_{h k} b^{h} b^{k}\right\}\left(q^{2}-p^{2} b^{2}\right)\left(\frac{p q^{4} \lambda}{8 L^{2}}\right) \\
& \left.+\left(\frac{1}{p} E_{h k} g^{h k}-2 p q \lambda E_{h k} b^{h} b^{k}\right)\right], \\
\mu^{*}= & \mu \\
A_{i j}^{*}=- & 2 q\left[\rho-\left(\frac{1}{p} C_{m} b^{m}-2 p q \lambda C_{h k} b^{h} b^{k}\right) \frac{p^{2} q^{2} \lambda}{4 L}\right. \\
& -\left\{\frac{1}{p}(n-3)-2 p q \lambda h_{h k} b^{h} b^{k}\right\}\left(q^{2}-p^{2} b^{2}\right)\left(\frac{p q^{4} \lambda}{8 L^{2}}\right) \\
+ & \left.\left(\frac{1}{p} E_{h k} g^{h k}-2 p q \lambda E_{h k} b^{h} b^{k}\right)\right] m_{i} m_{j} \\
+ & \mu\left\{M\left(m_{i} C_{j}+m_{j} C_{i}\right)+2 p^{2} q \lambda\left(C_{i} C_{j .}+C_{j} C_{i .}\right)\right. \\
& -M^{2} m_{i} m_{j}-2 p^{2} q \lambda M\left(m_{i} C_{j .}+m_{j} C_{i .}\right) \\
& \left.-4 p^{4} q^{2} \lambda^{2} C_{i .} C_{j .}\right\}+\left(\frac{1}{p} C_{m} b^{m}-2 p q \lambda C_{h k} b^{h} b^{k}\right) \\
& \left(\frac{p^{2} \lambda}{L} m_{i} m_{j}-p q^{3} C_{i j}\right)+\left\{\frac{(n-3)}{p}-2 p q \lambda h_{h k} b^{h} b^{k}\right\} \\
& {\left[\frac{p q^{4} \lambda}{4 L^{2}}\left\{q^{2}\left(2 p^{3}+1\right)-2 p^{5} b^{2}\right\} m_{i} m_{j}-\frac{p^{2} q^{2} \lambda}{4 L} C_{i j}\right] }
\end{aligned}
$$




$$
\begin{aligned}
& +\frac{1}{p}\left(C_{i j} g^{h k} d_{h k}-C_{i m}^{h} b^{m} d_{h j}-C_{j m}^{k} b^{m} d_{i k}\right) \\
& -2 p q \lambda\left(p S_{h i j k}+C_{i j} d_{h k}-C_{i k} d_{h j}-C_{h j} d_{i k}\right. \\
& \left.-h_{i k} E_{h j}-h_{h j} E_{i k}\right) b^{h} b^{k}
\end{aligned}
$$

Therefore, we have the following:

Theorem 4.1. An S-reducible Finsler space is transformed to an S-reducible Finsler space under the transformation (4.1) if and only if

$$
A_{i j}^{*}=0 \text {. }
$$

\section{The transformation of $S$-reducible Finsler space by one Form}

Let $\left(F_{n}, L^{*}\right)$ be a Finsler space obtained from a Finsler space $\left(F_{n}, L\right)$ by the transformation

$$
L^{* 2}(x, y)=L^{2}(x, y)+\beta^{2}
$$

where $\beta=b_{i}(x) y^{i}$ is of form in $\left(F_{n}, L\right)$. Such a transformation was fiest introduced by M. Matsumoto ([1]). The contravariant component of metric tensor $g^{* i j}$ of $\left(F_{n}, L^{*}\right)$ has been given as

$$
g^{* i j}=g^{i j}-\frac{1}{1+b^{2}} b^{i} b^{j}
$$

where $b^{i}=g^{i j} b_{j}$ and $b^{2}=g^{i j} b_{i} b_{j}$

We also gave the $(h) h v$-torsion tensor $C_{j k}^{* i}$ of $\left(F_{n}, L^{*}\right)$ as

$$
C_{j k}^{* i}=C_{j k}^{i}-\frac{1}{1+b^{2}} C_{. j k} b^{-1}
$$

where $C_{. j k}=C_{i j k} b^{i}$

By using the above relation, he obtained a relation between $v$-curvature tensors of $\left(F_{n}, L\right)$ and $\left(F_{n}, L^{*}\right)$ as

$$
S_{i j k l}^{*}=S_{i j k l}+\frac{1}{1+b^{2}}\left(C_{. i k} C_{. j l}-C_{. i l} C_{. j k}\right)
$$


With the help of equations (5.3) and (1.4), we get

$$
C_{i} C_{j}=C_{i}^{*} C_{j}^{*}-\frac{C_{. . i} C_{. . j}}{\left(1+b^{2}\right)^{2}}+\left(C_{i} C_{. . j}+C_{j} C_{. . i}\right) /\left(1+b^{2}\right)
$$

From (5.1), we get the angular metric tensor $h_{i j}^{*}=L^{*} \frac{\partial^{2} L^{*}}{\partial y^{i} \partial y^{j}}$ of $\left(F_{n}, L^{*}\right)$ as

$$
h_{i j}^{*}=h_{i j}+\frac{\beta^{2}}{L^{* 2}} l_{i} l_{j}+\frac{L^{2}}{L^{* 2}} b_{i} b_{j}+\frac{L \beta}{L^{* 2}}\left(l_{i} b_{j}+l_{j} b_{i}\right)
$$

In view of equations (1.2), (1.3), (5.2) and (5.4), the $v$-Ricci tensor is obtained as given below:

$$
\begin{aligned}
S_{i j}^{*}= & \rho h_{i j}+\mu C_{i} C_{j}+\frac{1}{1+b^{2}}\left(C_{. j}^{k} C_{. j k}-C . C_{. i j}\right) \\
& -\frac{1}{1+b^{2}}\left[S_{. i j .}+\frac{1}{1+b^{2}}\left(C_{. . j} C_{. . i}-C_{. . .} C_{. i j}\right)\right]
\end{aligned}
$$

Using equations (5.5) and (5.6), equation (5.7) can be written as

$$
S_{i j}^{*}=\rho^{*} h_{i j}^{*}+\mu^{*} C_{i}^{*} C_{j}^{*}+B_{i j}^{*}
$$

where $\rho^{*}=\rho$ and $\mu^{*}=\mu$ and

$$
\begin{aligned}
S_{i j}^{*}= & \rho\left\{\frac{\beta^{2}}{L^{* 2}} l_{i} l_{j}+\frac{L^{2}}{L^{* 2}} b_{i} b_{j}+\frac{L \beta}{L^{* 2}}\left(l_{i} b_{j}+l_{j} b_{i}\right)\right. \\
& +\mu \frac{C_{i} C_{. . j}+C_{j} C_{. . i}}{1+b^{2}}-\frac{C_{. i .} C_{. . j}}{\left(1+b^{2}\right)^{2}}+\frac{1}{1+b^{2}}\left(C_{. j}^{k} C_{. i k}-C_{. C} C_{. i j}-S_{. i j .}\right) \\
& -\frac{1}{\left(1+b^{2}\right)^{2}}\left(C_{. . j} C_{. . i}-C_{. . .} C_{. i j}\right.
\end{aligned}
$$

Therefore, we have the following:

Theorem 5.1. An S-reducible Finsler space is transformed to an $S$-reducible Finsler space under the tranaformation (5.1) if and only if

$$
B_{i j}^{*}=0
$$


6. The transformation of $S$-reducible Finsler space by $\beta$-change

Let $\left(F_{n}, L^{*}\right)$ be a Finsler space obtained from a Finsler space $\left(F_{n}, L\right)$ by the transformation ([1])

$$
\mathbb{L}^{*}(x, y)=\mathbb{L}(x, y)+\beta(x, y)
$$

where $\beta=b_{i}(x) y^{i}$.

The contravariant component of metric tensor has been given as

$$
g^{* i j}=L L^{*-1} g^{i j}-L L^{*-2}\left(y^{i} b^{j}+y^{j} b^{i}\right)+\left(L b^{2}+\beta\right) L^{*-3} y^{i} y^{j}
$$

where $b^{i}=g^{i j} b_{j}$ and $b^{2}=g^{i j} b_{i} b_{j}$

The $(h) h v$-torsion tensor $C_{j k}^{* i}$ of $\left(F_{n}, L^{*}\right)$ has been given as

$$
\begin{aligned}
C_{i k}^{* j}= & C_{i k}^{j}-\frac{1}{2} L^{*-1}\left(h_{i}^{j} m_{k}+h_{k}^{j} m_{i}+h_{i k} m^{j}\right) \\
& -\left\{L^{*-1} C_{. i k}-\frac{1}{2} L^{*-2}\left(m h_{i k}-2 m_{i} m_{k}\right)\right\} y^{j}
\end{aligned}
$$

where $h_{i j}=g_{i j}-L^{-2} y_{i} y_{j}, m_{i}=\beta L^{-2} y_{i}-b_{i}$

The $v$-curvature tensor of $\left(F_{n}, L^{*}\right)$ has been given as

$$
\begin{aligned}
S_{i j k l}^{*}= & L^{*} L^{-1} S_{i j k l}+\frac{1}{4} m^{2}\left(L^{*} L^{-1}\right)\left(h_{i l} h_{j k}-h_{i k} h_{j l}\right) \\
& +\frac{1}{2} L^{-1}\left(h_{i l} C_{. j k}-h_{i k} C_{. j l}+h_{j k} C_{. i l}-h_{j l} C_{. i k}\right) \\
& +\frac{1}{4}\left(L^{*} L\right)^{-1}\left(h_{i l} m_{j} m_{k}-h_{i k} m_{j} m_{l}+h_{j k} m_{i} m_{l}-h_{j l} m_{i} m_{k}\right)
\end{aligned}
$$

where $m^{2}=g_{i j} m^{i} m^{j}$

With the help of equations (1.4) and (6.3), we get

$$
C_{i} C_{j}=C_{i}^{*} C_{j}^{*}+\left(\frac{n+1}{2}\right) L^{*-1}\left(C_{i} m_{j}+C_{j} m_{i}\right)-\left(\frac{n+1}{2}\right)^{2} L^{*-2} m_{i} m_{j}
$$

From (6.1), we have the following relation between angular metric tensor of $\left(F_{n}, L\right)$ and $\left(F_{n}, L^{*}\right)$

$$
h_{i j}=\left(\frac{L}{L+\beta}\right) h_{i j}^{*}
$$


In view of equation (1.2) and using also the relations (6.2), (6.4), (1.3), we have

$$
S_{i j}^{*}=(\rho+A) h_{i j}+\mu C_{i} C_{j}+B C_{i j}+D b_{i} b_{j}
$$

where

$$
\begin{aligned}
& A=\frac{1}{4}\left\{m^{2} L^{*-2}(n-2)+\frac{1}{2} L^{-1} C .+\frac{1}{4} L^{-2} m^{2}\right\} \\
& B=\frac{1}{2} L^{*-1}(n-3) \text { and } D=\frac{1}{4} L^{-2}(n-3)
\end{aligned}
$$

Using equation (6.5), (6.6) and (6.7), the $v$-Ricci tensor of $\left(F_{n}, \mathbb{L}^{*}\right)$ is given as

$$
S_{i j}^{*}=\rho^{*} h_{i j}^{*}+\mu^{*} C_{i}^{*} C_{j}^{*}+D_{i j}^{*}
$$

where

$$
\rho^{*}=\left(\frac{\rho+A}{L+\beta}\right) L, \quad \mu^{*}=\mu
$$

and

$$
D_{i j}^{*}=\frac{\mu}{2}(n+1) L^{*-1}\left(C_{i} m_{j}+C_{j} m_{i}\right)+\left\{D-\mu\left(\frac{n+1}{2}\right)^{2} \mathbb{L}^{*-2}\right\} m_{i} m_{j}+B C_{i j}
$$

Therefore, we have the

Theorem 6.1. An S-reducible Finsler space is transformed to an $S$-reducible Finsler space under the transformation (6.1) if and only if

$$
D_{i j}^{*}=0 \text {. }
$$

\section{References}

[1] Matsumoto, M., "On some transformations of locally minkowskian spaces", Tensor, $N$. S., Vol. 22 (1971), 103-111.

[2] Matsumoto, M., "On Finsler spaces with curvatures of some special forms", Tensor, 22 (1971), 201-204.

[3] Matsumoto, M., "On C-reducible Finsler space", Tersor N. S., Vol. 24 (1972), 29-37.

[4] Matsumoto, M. and Shibata, C., "On semi- $C$ reducibility", $T$-tensor $=0$ and $S 4$-likensess of Finsler spaces", J. Math. Kyoto Univ. 19 (1979), 301-314.

[5] Matsumoto, M. and Numata, S., "On semi $C$-reducible Finsler spaces with constant coefficients and $C_{2}$-like Finsler spaces", Tersor, N. S., 34 (1980) 218-222.

[6] Prasad, B. N. and Singh J. N., "Cubic transformations of Finsler spaces and $n$-fundamental forms of their hypersurfaces", Indian J. pure appl. Math., 20(3), March 1989, 242-249.

[7] Prasad, B. N. and Srivastava, L., "A transformation of the Finsler metric by an $h$-vector", India J. pure appl. Math., 20(5), May 1989, 455-465. 
[8] Takano, Y., "On theory of Fields in Finsler spaces", (Proc. Intern. Symp. Relativity and Unified Field theory, Calcutta, 1975-76, 17-26 (M5B(3042)) 20245.

[9] Takano, Y., "Gange Fields in Finsler spaces" (Lett. Nuovo Cimento 35 (1982), 213-217 (M84 m (5089) 53079.

Department of Mathematics, St. Andrew's College, Gorakhpur, India.

Department of Mathernatics, University of Grakhpur, Gorakhpur, India. 